\title{
Lung Development in the Fetal Primate, Macaca nemestrina. II. Pressure-Volume and Phospholipid Changes
}

\author{
SUSAN PALMER, THOMAS E. MORGAN, JOHN L. PRUEITT, JANET H. MURPHY, \\ AND W. ALAN HODSON ${ }^{(43)}$ \\ Departments of Pediatrics and Medicine, Regional Primate Research Center, and Child Development and \\ Mental Retardation Center, University of Washington, Seattle, Washington, USA
}

\section{Summary}

The biological and physiologic maturation of the lung in the primate Macaca nemestrina (pigtail monkey) from 107 days of gestation through term is the subject of this report. Total lung volume increased approximately $160 \%$ during the last $20 \%$ of gestation (Fig. 1). The increase from $30 \%$ to $85 \%$ of total lung volume at a deflation pressure of $10 \mathrm{~cm} \mathrm{H}_{2} \mathrm{O}$ indicates a marked change in lung stability during the last 30-40 days of gestation (Fig. 2). Lung phospholipid per $g$ dry weight of lung more than doubles during the last $20 \%$ of gestation (Fig. 3). This increase in phospholipid is due almost entirely to an increase in lecithin, and surface active material (SAM) lecithin accounts for the major part of this increase (Figs. 4 and 5). The increases in total lung and SAM lecithin parallel but precede the increase in lecithin in amniotic fluid (Fig. 6). As lung SAM increases the amniotic fluid lecithin to sphingomyelin ratio also increases (Fig. 7 and Table 1). Low ratios of lecithin to protein in SAM are found before 135 days of gestation. Subsequently, the amount of lecithin increases and, although protein also increases, the ratio increases 4-fold (Fig. 8). The amount of lung required to reduce surface tension of $1 \mathrm{~cm}^{2}$ to 12 dynes tended to decrease with advancing gestational age (Fig. 9 and Table 1). Parallel studies of airway generation demonstrate a similarity to the human fetal lung. Thus, the structural, compositional, and physiologic changes described in our studies strongly support the use of the fetal monkey for studies of developmentally related disorders of the human lung.

\section{Speculation}

Biochemical and functional maturational changes in the fetal $M$. nemestrina indicate its suitability for studies of developmentally related pulmonary disorders of the human. Premature delivery by cesarean section between 130 and 140 days should provide a satisfactory homolog for hyaline membrane disease.

Body and lung growth in the primate $M$. nemestrina in the last third of gestation has been described in a previous paper (19). The biochemical and physiologic maturation of the lung in this primate from 107 days of gestation through term (168 days) is the subject of this report. Boyden (3) has shown that development of the terminal air sacs and airways in $M$. nemestrina closely resembles that of the human. Maturational events in the primate $M$. nemestrina are presented, demonstrating the suitability of this primate for studies of human fetal lung development and clinically associated abnormalities.

\section{MATERIALS AND METHODS}

The lungs of 23 fetal and 2 newborn $M$. nemestrina were obtained as previously described (19). In most cases, either the right upper lobe or the medial basal (rose) lobule was removed for microscopic studies. Static pressure volume curves were obtained from the remaining lung. Deflation volumes after the second inflation were recorded at intervals between 35 and $0 \mathrm{~cm}$ $\mathrm{H}_{2} \mathrm{O}$ transpulmonary pressure (4). Weights of the individual lobes were then obtained and samples were taken for determination of percentage of dry weight. The right and left lungs (upper and lower lobes combined) were homogenized separately in $0.145 \mathrm{M} \mathrm{NaCl}, 0.01 \mathrm{M}$ Tris, and 0.001 M EDTA, pH 7.4. Homogenization was usually completed within $30 \mathrm{~min}$ of time of death. Aliquots of the homogenates were taken for determination of protein, hemoglobin, and DNA (19), and the remainder of the homogenates was used immediately for lipid extraction and isolation of SAM.

Surface active materials were isolated from lung homogenate by a modification of the method of Frosolono et al. (13). An aliquot of the lung homogenate was layered over $10 \mathrm{ml}$ of 0.75 $M$ sucrose in homogenization solution and centrifuged at 48,000 $\times g$ for $40 \mathrm{~min}$ in a Sorvall SS-34 rotor at $0^{\circ}$. Material at the interface was carefully removed with a disposable pipette, washed in distilled $\mathrm{H}_{2} \mathrm{O}$, and centrifuged at $48,000 \times \mathrm{g}$ for 20 min. The washing and sedimentation were repeated and the sediment (designated SAM) was resuspended in water. The SAM thus isolated presumably represents materials from both intra- and extracellular compartments of the lung. Protein in SAM was measured by the method of Lowry (27). Qualitative and some semiquantitative determinations of the surface activity of SAM isolates were made on a modified Wilhelmy surface balance at room temperature (6). Aliquots of SAM were spread on clean Ringer solution, $\mathrm{pH} 7.4$, from a suspension of isopropyl alcohol-SAM, 1:1 (v/v) at room temperature. For the semiquantitative assays, the weight of lung required to yield the smallest aliquot of SAM which would reduce surface tension of 1 $\mathrm{cm}^{2}$ to 12 dynes/cm was calculated (6). This was divided by the surface area at 12 dynes $/ \mathrm{cm}$. The quotient is the surface concentration for lung weight and is inversely related to the amount of SAM present in the lung tested. Since this technique was developed during the progress of this study, data were obtained from only four animals.

Lipids were extracted from lung homogenate and from lung surface active material according to the method of Morgan and Edmunds (29), except that extracts were taken to volume in $2: 1$ $(\mathrm{v} / \mathrm{v})$ chloroform-methanol containing $0.05 \%$ butylhydroxytoluene (BHT) to prevent oxidative degradation of the lipids. Amniotic fluid was centrifuged at $250 \times \mathrm{g}$ for $5 \mathrm{~min}$. One volume of supernatant was added to $1.25 \mathrm{vol}$ methanol in a separatory funnel and mixed. After $20 \mathrm{~min} 1.25$ vol chloroform were added, the contents mixed again by shaking, and after 10 min another 1.25 vol chloroform were added. The phases were separated at $4^{\circ}$. The lower phase was removed to a round bottomed flask and the upper phase extracted with $2.5 \mathrm{vol}$ chloroform. The combined lower phases were dried in vacuo and 
stored at $-20^{\circ}$ in $2: 1$ chloroform-methanol containing $0.05 \%$ BHT.

The lipid extracts were analyzed for phosphorus by the Bartlett method (2), modified by using perchloric acid instead of sulfuric acid for digestion (7). Phospholipids were separated by thin layer chromatography on silica gel $\mathrm{H}$ (Merck-Darmstadt) in a solvent system of chloroform-methanol- $\mathrm{H}_{2} \mathrm{O}$ 65:25:4 (v/v). The spots were visualized with iodine vapor, identified by comparison with the migrations of known standards, scraped into tubes, and assayed directly for phosphorus (35). Minimum disaturated lecithin determinations were performed as follows: lecithin was separated from the total lipid extract by thin layer chromatography on silica gel HR, identified with dichlorofluorescein and ultraviolet light, and scraped into centrifuge tubes. The gel was eluted three times with $2 \mathrm{ml}$ developing solvent. An aliquot of the eluted lecithin was set aside for determination of total lecithin fatty acids. The remainder was dried under $\mathrm{N}_{2}$ and treated with Naja naja venom according to the method of Long and Penny (28). After hydrolysis the ether layer was removed and the aqueous layer was extracted with $6 \mathrm{ml} \mathrm{2:1} \mathrm{chloroform-}$ methanol. The lower chloroform layer was combined with the ether extract, dried under $\mathrm{N}_{2}$, and rechromatographed on silica gel HR. This chromatogram also served as a check on the completeness of hydrolysis of the lecithin. The lysolecithin and free fatty acids were visualized, scraped, and eluted. Methanolysis of total lecithin, lysolecithin, and free fatty acids was performed by the method of Ways et al. (39). The hexane extract was washed once with $10 \mathrm{ml} \mathrm{H}_{2} \mathrm{O}$ before being concentrated and injected on a Barber-Colman 5000 gas chromatograph. The percentage of distribution of individual fatty acids derived from the total (nonhydrolized) lecithin and from the hydrolysis products were compared by the formula:

$\%$ Fatty acid $=(\%$ fatty acid in $\alpha+\%$ fatty acid in $\beta$ position $) / 2$

This comparison served to check on possible degradative changes to fatty acids during hydrolysis or subsequent chromatographic separation. Agreement within $\pm 10 \%$ was required for acceptance of these data for calculation of minimum disaturated lecithin. Minimum disaturated lecithin was determined from the product: (\% $\alpha$ saturated fatty acid) $-(\% \beta$ unsaturated fatty acid) $\times(\mathrm{mg}$ lecithin $)$.

\section{RESULTS}

Pressure-volume curves were obtained in 19 fetal animals and 1 newborn. Deflation curves were markedly different for the animals over the range of ages studied. For clarity in presentation the animals are grouped by gestational age: immature (115130 days), transitional (135-149 days), and mature (153-168 days) (Fig. 1). The mean deflation volumes at $10 \mathrm{~cm} \mathrm{H}_{2} \mathrm{O}$ airway pressure are $3.8,16.1$, and $23.4 \mathrm{cc} / \mathrm{g}$ dry weight, respectively. These differences at $10 \mathrm{~cm} \mathrm{H}_{2} \mathrm{O}$ pressure were significant, comparing immature with the transitional group, $P<0.001$, and transitional with the mature group, $P<0.05$. The groups were not significantly different at zero pressure; the mean volumes were $0.3,3.4$, and $7.1 \mathrm{cc} / \mathrm{g}$ dry lung, respectively. The deflation characteristics, expressed as percentage of total lung volume are illustrated in Figure 2. The percentages of maximum lung volume at $10 \mathrm{~cm} \mathrm{H} \mathrm{H}_{2} \mathrm{O}$ pressure for the three groups are 30,71 , and $85 \%$, respectively, and these differences were significant, comparing immature with the transitional group, $P<0.001$, and transitional with the mature group, $P<0.025$. The deflation curve for the one newborn animal ( $72 \mathrm{hr}$ postnatal age) was identical to the curve of the mean values of percentage maximum volume for the mature fetal group. Its lungs contained about one-fourth more air at maximum volume than the mean of the mature fetal group. The largest maximum lung volume occurred in a fetal animal of 160 days of gestation.

Lung phospholipid per $\mathrm{g}$ dry weight of lung more than doubles during the last $20 \%$ of gestation (Fig. 3). This increase in phospholipid is almost entirely due to the increase in lecithin,

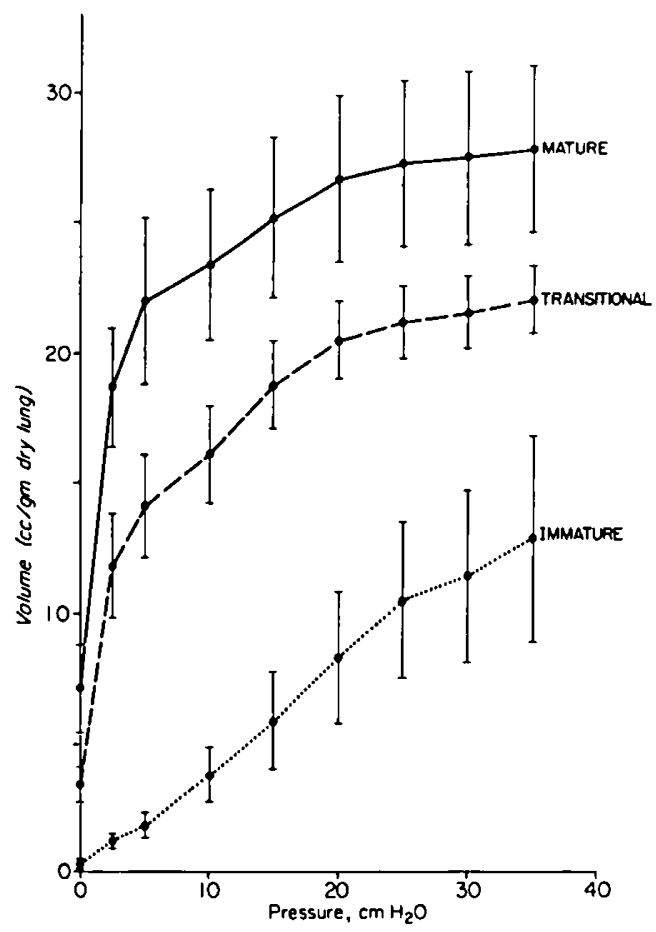

Fig. 1. Deflation pressure-volume relationships in $M$. nemestrina Values are mean \pm SEM. The upper curve represents one newborn and six fetal animals (153 days to term); the middle curve, eight animals (135-149 days) and the lower curve, five animals (115-130 days).

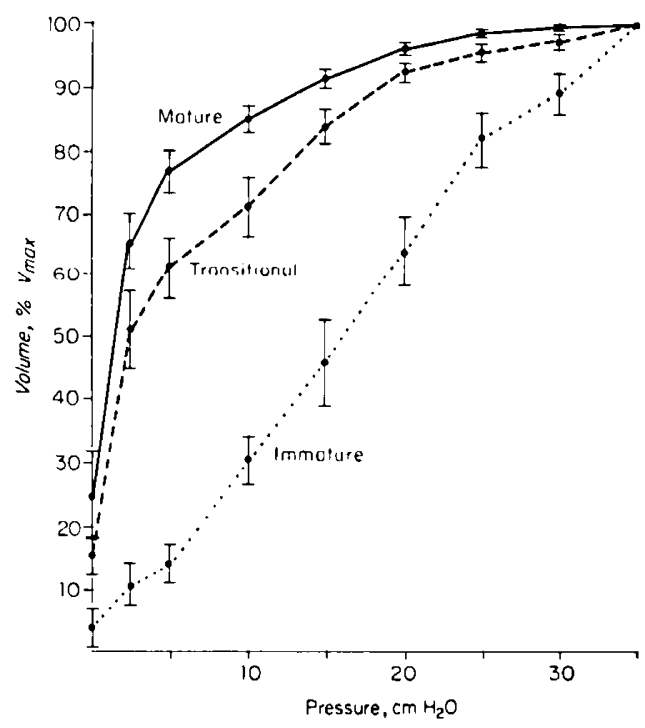

Fig. 2. Deflation pressure-volume curves. Values are mean \pm SEM. Animals are grouped as in Figure 1.

which accounts for almost $70 \%$ of the total phospholipid at term, but for only $45 \%$ of the total phospholipid before 135 days gestation. Minimum disaturated lecithin increases from $10 \%$ of the total phospholipid (22\% of the lecithin) before 135 days to $29 \%$ of the total phospholipid ( $44 \%$ of the lecithin) at term. The most striking increase occurs in the SAM lecithin (Figs. 4 and 5), which comprises $4 \%$ of the total phospholipid before 135 days and $33 \%$ of the total phospholipid at term: a 16-fold increase in the amount of SAM lecithin per $g$ dry weight of lung. The increase in lung lecithin is due almost entirely to the increase in SAM lecithin.

Linear regression analysis of SAM phosphatidylcholine and \% $\mathrm{V}_{\max }$ at $\mathrm{P}_{10}$ yields a correlation coefficient of $0.93\left[\mathrm{~V}_{\max }=\right.$ $14.0+19.0$ (log SAM phosphatidylcholine)]. Amniotic fluid 


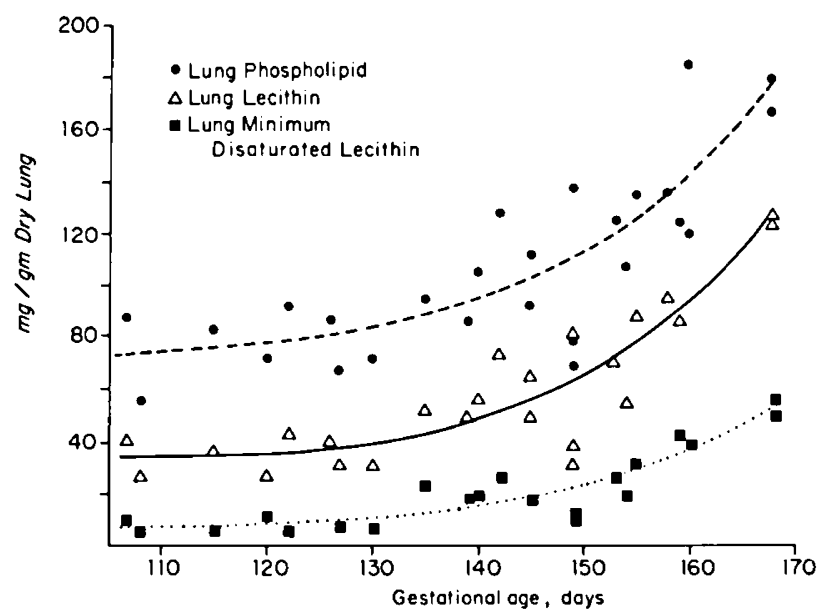

Fig. 3. Relationship of lung phospholipid, lecithin and minimum disaturated lecithin to fetal age. Each regression curve is a fit to the least squares of the following equations: phospholipid $=77.4+[0.064$ (age) $]$ $+1.76 \times 10^{-14}(\text { age })^{\mathrm{s}}$; lecithin $=60.9+[0.277$ (age) $]+1.79 \times 10^{-14}$ (age $)^{8}$ minimum disaturated lecithin $=12.1+[0.058$ (age) $]+0.80 \times$ $10^{-14}(\text { age })^{4}$.

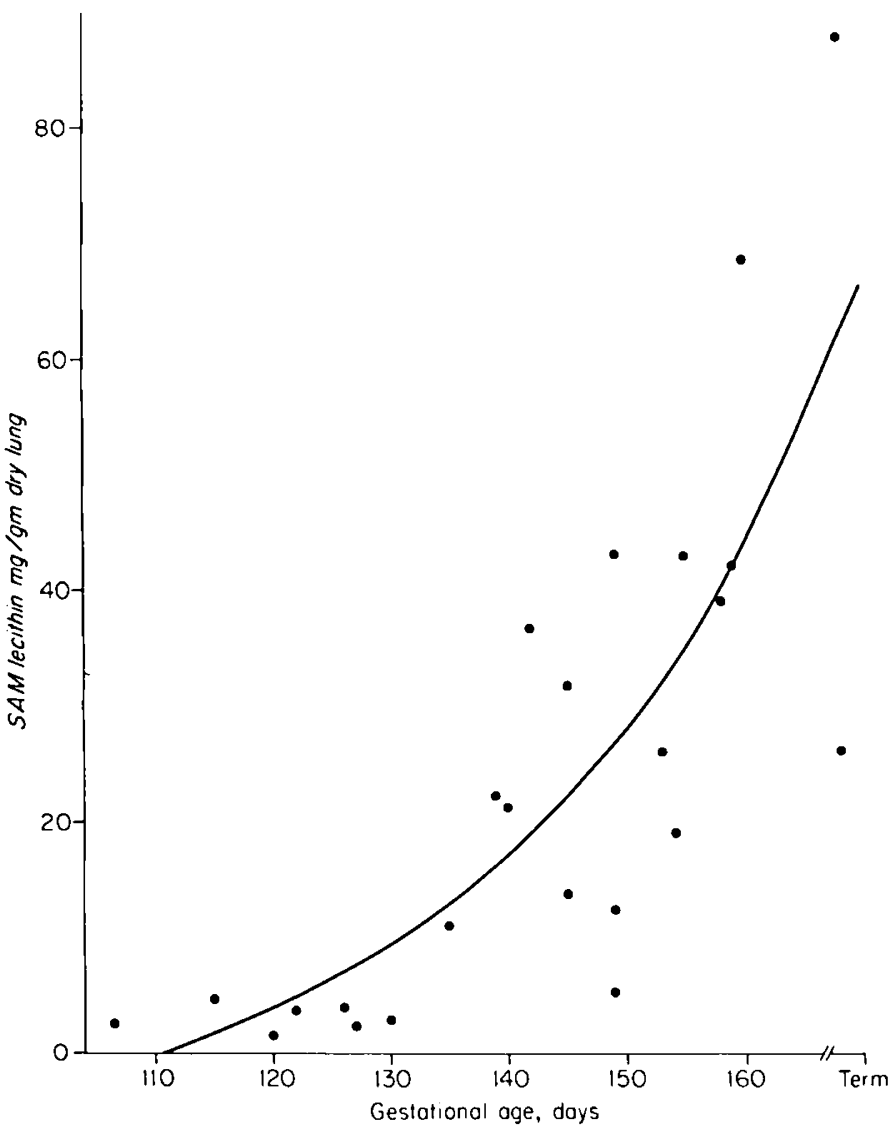

Fig. 4. Relationship of lung surface active material (SAM) lecithin to fetal age. The regression curve is fit to the least squares of the equation: SAM lecithin $=30.1+0.258(\mathrm{age})+0.75 \times 10^{-14}(\text { age })^{\mathrm{x}}$.

lecithin increases after 130 days and at each gestational age, amniotic fluid lecithin is lower than lung lecithin (Fig. 6). As lung SAM increases, the ratio of lecithin-sphingomyelin $(\mathrm{L} / \mathrm{S})$ lipid phosphorus in amniotic fluid also increases (Fig. 7 and Table 1). The ratio of lecithin to protein in SAM isolated by density gradient centrifugation is shown in Figure 8. Very low values are found before 135 days of gestation. Subsequently, the

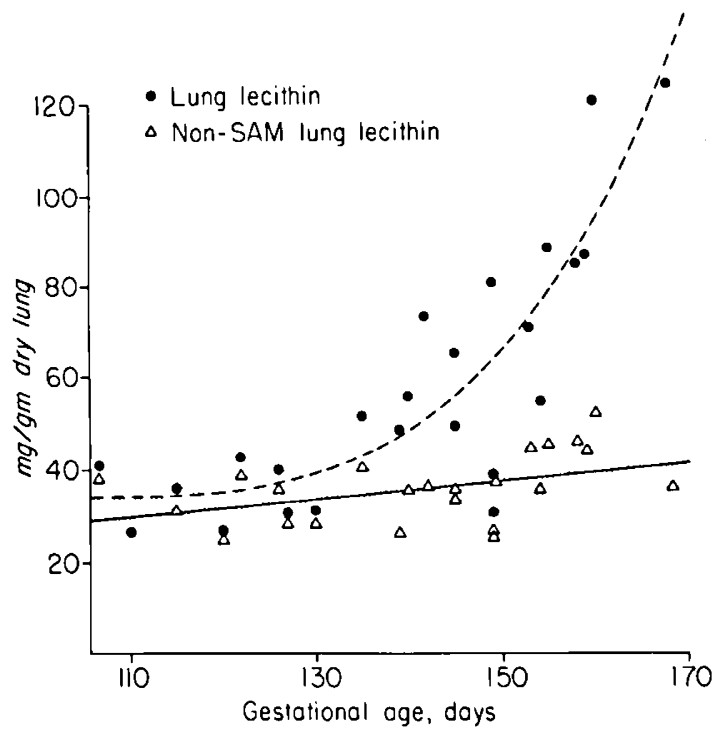

Fig. 5. Total lung lecithin increases sharply after 135 days of gestation. In contrast, non-surface active material (non-SAM) lecithin, derived by subtracting SAM lecithin from total lung lecithin, increases only slightly with advancing gestation.

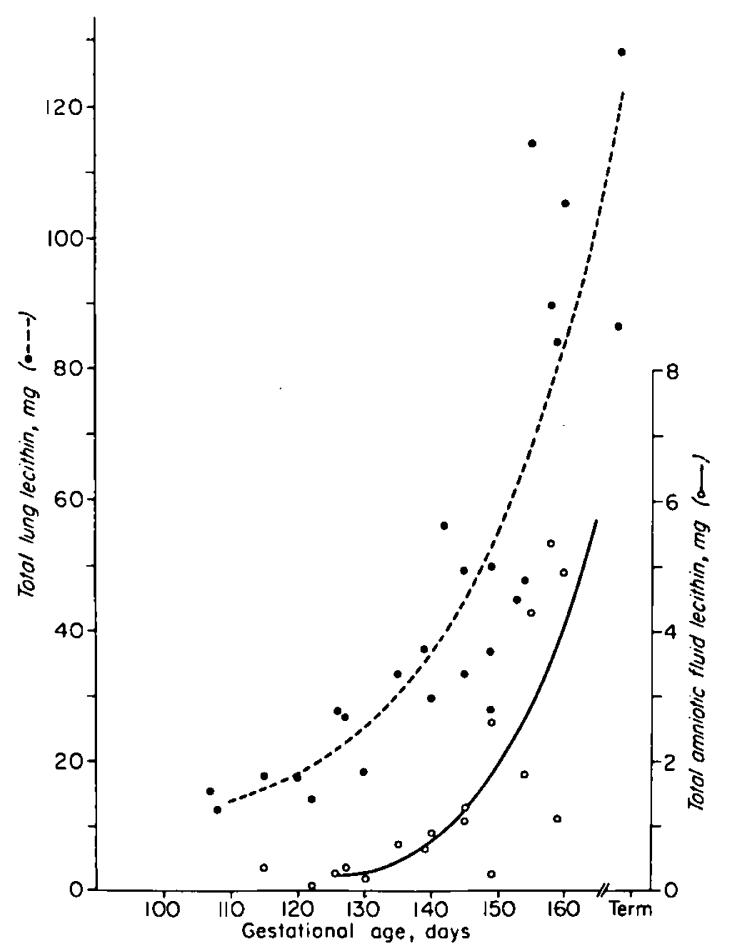

Fig. 6. The relationship of total lung lecithin to total amniotic fluid lecithin (amniotic fluid volume is assumed to be $100 \mathrm{ml}(38)$. The regression lines are fit to the least squares of the equations: total lung lecithin $=1.99+0.078$ (age) $+1.61 \times 10^{-14}(\text { age })^{8} ;$ amniotic fluid lecithin $=6.83+[0.061($ age $)]+0.00163 \times 10^{-14}(\text { age })^{8}$.

amount of lecithin increases and, although protein also increases, the ratio increases 4 -fold.

Qualitative surface tension area measurements were made on SAM isolated from 14 animals (Table 1, Fig. 9). Minimum surface tensions were 12 dynes or less in one of three animals in the immature group, four of five animals in the transitional group, and six of six animals in the mature group. We were also able to apply a semiquantitative method developed late in the course of this study to four of these animals. Surface concentrations at 145,149, and 159 days of gestational age and in a 
newborn animal at term (168 days) were $31,160,17$, and $20 \mu \mathrm{g}$ dry lung/ $\mathrm{cm}^{2}$ at 12 dynes, respectively (Table 1 ). The value of $160 \mu \mathrm{g}$ lung $/ \mathrm{cm}^{2}$ in a 149 -day animal appears to be disparate. However, SAM lecithin, percentage of $V_{\max }$ at $P_{10}$, and amniotic

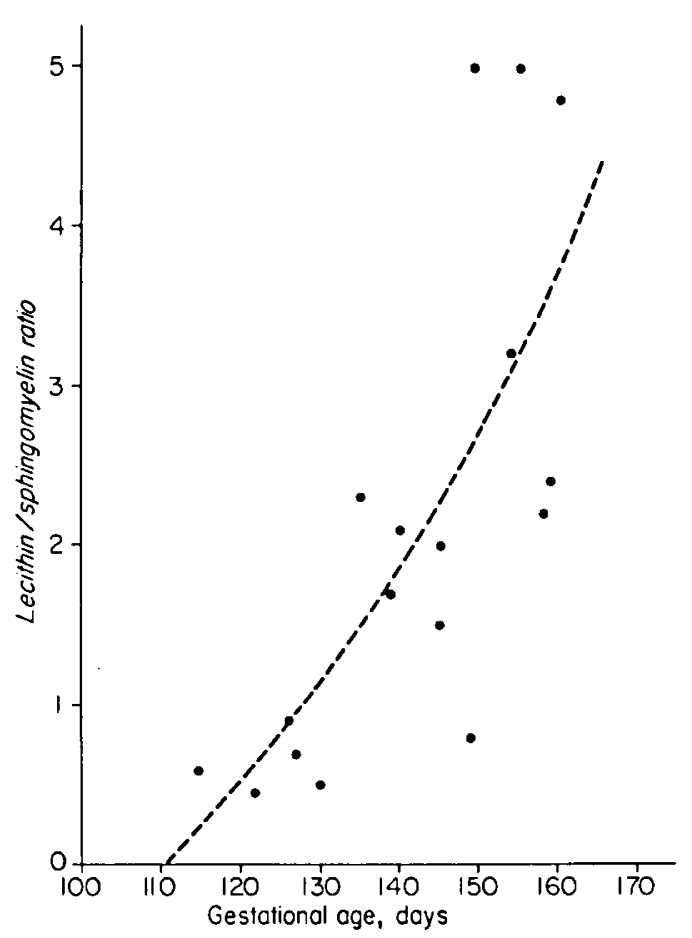

Fig. 7. Lecithin sphingomyelin (L/S) ratios in amniotic fluid plotted against gestational age. The regression line is fit to the least squares of the equation: $\mathrm{L} / \mathrm{S}$ ratio $=5.70+0.051$ (age) $+0.031 \times 10^{-14}(\text { age })^{8}$. fluid $\mathrm{L} / \mathrm{S}$ ratio were also consistently low in this animal (Table 1).

\section{DISCUSSION}

The development of volume stability, the increase in lung phospholipids, and the differentiation of type II alveolar epithelial cells are events necessary for successful adaptation of the fetal lung to air breathing. Prior studies in other species have focused on the development of pressure-volume stability of fetal lungs and surface activity of fetal lung extracts. Initial studies in the mouse (5) suggested that surfactant appeared suddenly at about $90 \%$ of gestation. Similarly, rapid increases have been described in the rat (38) and rabbit (18), occurring at about $87 \%$ of gestation. More extensive studies have been completed in the fetal lamb which describe an increase in lung phospholipid (4), appearance of the osmiophilic inclusion bodies in type II alveolar epithelial cells $(23,34)$, an increased surface activity of lung extracts (4), pressure-volume stability (4), and increased amniotic fluid lecithin (37) with advancing gestational age.

Data from human studies $(17,20)$ suggest that changes in pulmonary phospholipids and maturity occur between $70 \%$ and $80 \%$ of gestation. The results of studies of human lungs obtained at autopsy from abortuses on infants are difficult to interpret because of autolytic changes occurring between death and the time when tissue is available for analysis. In attempts to obtain an experimental animal model more comparable to the human and to avoid the problems associated with human studies, investigators have recently studied the rhesus monkey. Morphologic studies $(3,22)$ suggest similarities between the monkey and the human. Kerr and Helmuth (21) have demonstrated an increase in palmitic acid on phosphatidylcholine between 125 and 150 days with a more marked rise after 150 days. Studies by Epstein et al. $(9,10)$ and Farrell et al. (12) show an increase in lecithin beginning at about 140 days of gestation consistent with the present study. Choline incorporation into lecithin increased between 148 and 153 days $(8,12)$. The changes in lung lecithin,

Table 1. Various parameters measured in 25 Macaca nemestrina ${ }^{1}$

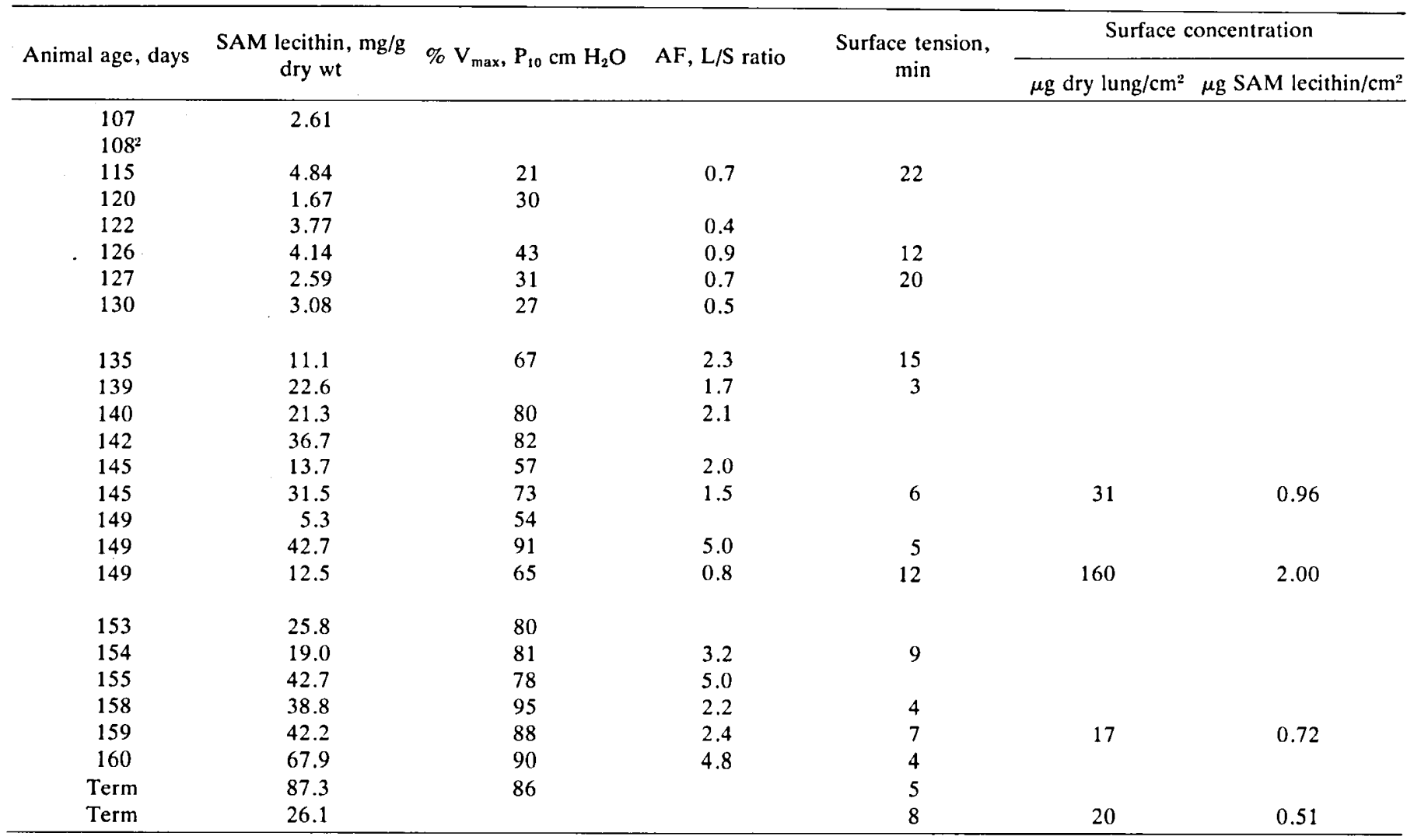

' SAM: surface active material; AF: amniotic fluid.

${ }^{2}$ No SAM isolate was attempted on this animal. 


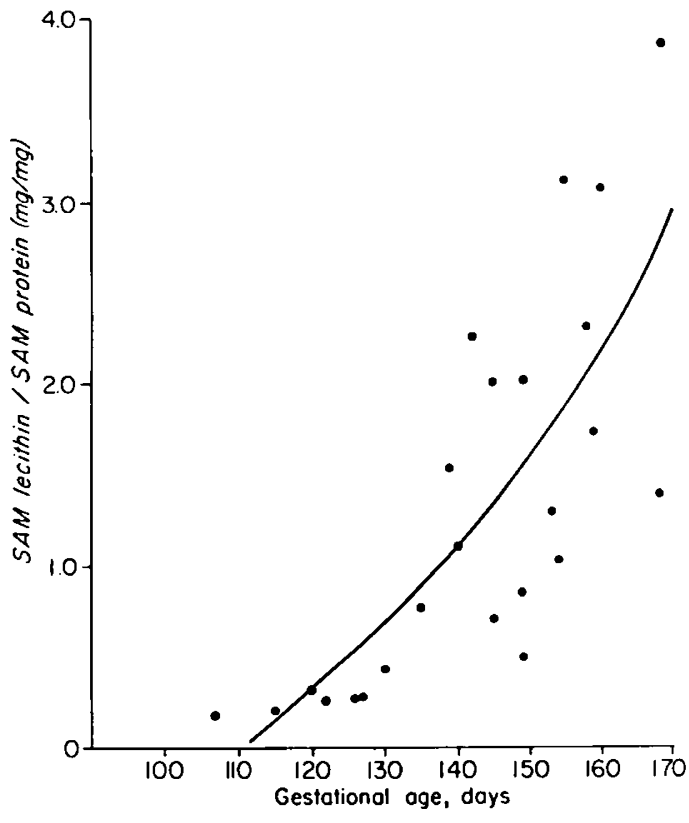

Fig. 8. Ratio of surface active material $(S A M)$ lecithin to SAM protein plotted against advancing gestational age. The regression line is fit to the least squares of the equations: SAM lecithin/SAM protein $=-3.32+0.029$ (age) $+0.018 \times 10^{-14}(\text { age })^{8}$.

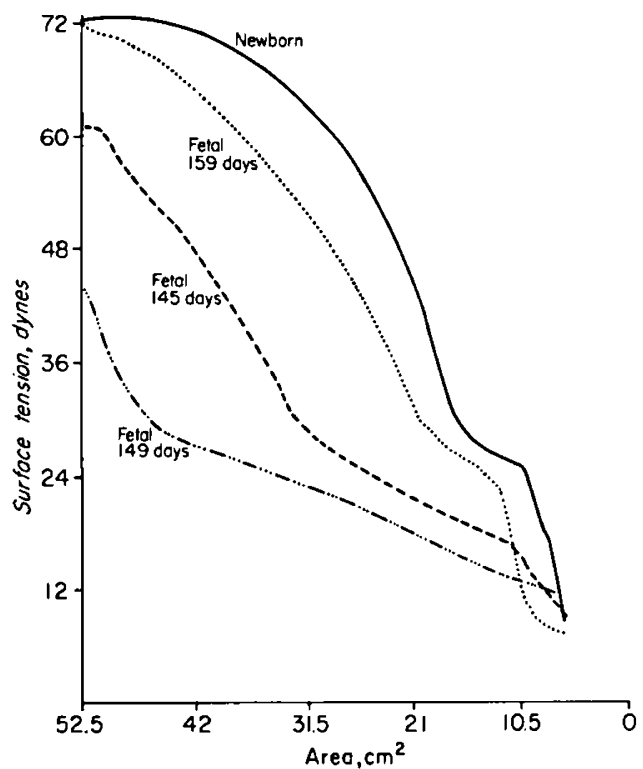

Fig. 9. Surface tension area measurements of surface active material isolated from four animals at the ages indicated.

therefore, appear to occur between $75 \%$ and $85 \%$ of term, more closely resembling the human than previously studied mammalian species. Gluck et al. (14) have compared L/S ratios in the baboon to the rhesus monkey and conclude that the monkey more closely resembles the human. Kling (26), however, demonstrated changes in baboon amniotic $\mathrm{L} / \mathrm{S}$ ratios comparable to that seen in the human $(15,16)$. There is no evidence at present to suggest any major differences between the two Macaca species, mulatta and nemestrina, in fetal lung development.

Modeling studies of airway generation by our colleague, Boyden (3), show that airway development of $M$. nemestrina is similar to the human fetal lung. Since airway development is complete prior to 110 days, proliferation of the terminal air sacs or potential "alveolar" development would be expected over the last third of gestation. The pressure-volume curves in this study
(Fig. 1) describe an increase in total lung volume of approximately $100 \%$ during the last $20 \%$ of gestation. In addition to the increase in total lung volume, there is a marked change in the stability of the lung, as seen in Figure 2. For example, the increase from $30 \%$ to $85 \%$ of total lung volume at a deflation pressure of $10 \mathrm{~cm} \mathrm{H}_{2} \mathrm{O}$ pressure suggests that development of stable air spaces occurs during the last 30-40 days of gestation. This process may be largely completed by 150 days of gestation. Development of alveoli in the terminal clusters or "racemes" requires an increase in the number of epithelial cells as well as differentiation of these cells. Histologic studies, including electron microscopy, of changes associated with alveolar development will be the subject of a separate report (31).

We have demonstrated in these experiments in the $M$. nemestrina fetus that phospholipids, lecithin, and minimum disaturated lecithin increase in the developing lung (Fig. 3), with a rapid increase in lung lecithin during the last 30-40 days of gestation. This suggests that either cell membrane lecithin continues to increase, or more likely, that the biosynthesis and/or storage of surfactant is increasing rapidly. The latter hypothesis is consistent with microscopic evidence that lamellated organelles appear in the type II alveolar epithelial cells late in gestation $(23,31)$. This hypothesis, particularly with respect to the increase in the saturated lecithin content of lung, is also consistent with the increase in stability of the lung described above.

The method used to estimate SAM in this study has limitations. The materials were recovered from lung homogenates and presumably represent SAM from both the intra- and extracellular locations. A compartmental distribution of SAM and changes in that distribution during gestation cannot be estimated from these data. Although lipids extracted from whole lung differ from lipids from airway lavage (30), SAM isolated from lung homogenate and airway lavage by density gradient and differential centrifugation $(24,36)$ is very similar in phospholipid composition and surface activity. The method used in this study yields materials which are enriched in disaturated phosphatidylcholine and lowers surface tension of an air-liquid interface to less than 10 dynes $/ \mathrm{cm}$. Also, the increase in SAM lecithin corresponds to the increase in volume stability during gestation. For these reasons we believe that this method allows us to recover a fraction representative of the surfactant complex and may be used to study changes during gestation.

It appears that the increase in total lung lecithin after 135 days is primarily due to the increase in lecithin in the SAM fraction (Figs. 4 and 5). When SAM lecithin was subtracted from total lung lecithin, the non-SAM lecithin in lung increased minimally with advancing gestational age (Fig. 5).

The increases in total lung and SAM lecithin parallel but precede the increase of lecithin in amniotic fluid (Fig. 6). This suggests that SAM is synthesized in lung and that storage and/or secretory events occur before SAM is released into the amniotic fluid from the future air spaces via the tracheal fluid. Our results are consistent with those of King et al. (25), who have demonstrated concomitant increases in SAM apoprotein and lipid in the amniotic fluid of the human infant. The increase in the ratio of lecithin to protein in SAM which we have demonstrated is consistent with the hypothesis that apoprotein is synthesized first by the lung cells and that the lipid components are then added to form the lipoprotein complex, which is later released into the tracheal fluid. A hypothetical sequence of events in the development of the surface active complex is illustrated in Figure 10. Prior to 120 days apoprotein may be present in low concentration at which time SAM lecithin and disaturated lecithin content is minimal. After 130 days of gestation the apoprotein-lecithin complex can be isolated and the amount of this complex increases throughout the last portion of gestation. The amount of lecithin in the amniotic fluid increases about 10 days later (after 140 days of gestation) and increases rapidly to term.

Description of the morphologic development (growth of airways, alveoli, and blood vessels) is lacking in other studies of fetal lung development. Thus, the structural, compositional, and physiologic changes described in our studies and those reported 


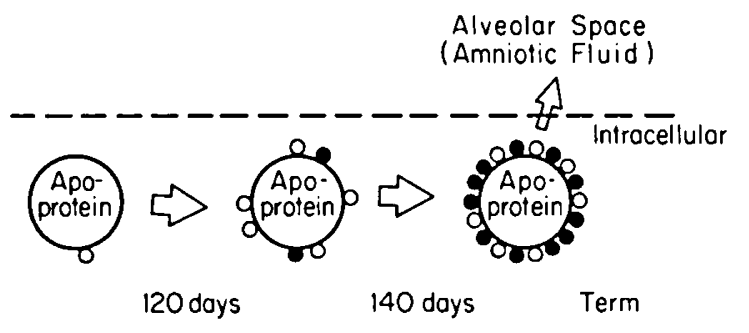

Fig. 10. A schematic representation of the hypothetical maturation of the surface active material lipoprotein complex. The small open circles represent unsaturated and the closed circles saturated phospholipids, principally phosphatidylcholine.

in the literature strongly support the use of the fetal monkey lung for studies of developmentally related disorders of the human lung. For example, although advances have been made in understanding the pathophysiology of hyaline membrane disease (11), information about sequential qualitative and quantitative changes in SAM from human infants has not been obtained. Studies on therapeutic measures such as prevention of premature delivery and pharmacologic acceleration of maturation (e.g., glucocorticoids) will require a satisfactory homolog for physiologic, biochemical, and ultrastructural studies of the effects of these agents on the immature lung and other systems. Preliminary studies in the next phase of our investigations have shown that the premature $M$. nemestrina delivered by cesarian section between 130 and 140 days develops hyaline membrane disease and serves as a satisfactory homolog for the human disease (32).

\section{CONCLUSION}

1. Physiologic and biochemical maturation of the fetal primate lung ( $M$. nemestrina) has been studied over the last onethird of gestation (term $=168$ days).

2. Lung volume increases $100 \%$ during the last $20 \%$ of gestation.

3. Lung pressure-volume stability increases markedly over this period.

4. There was a marked increase in lung phospholipid content, mainly due to an increase in lecithin, particularly SAM lecithin.

5. The rise in lecithin in amniotic fluid begins approximately 10 days after the increase in lung lecihtin. The increase in amniotic fluid lecithin is accompanied by an increase in the $\mathrm{L} / \mathrm{S}$ ratio.

6. The lecithin to protein ratio of SAM increases 4-fold after 135 days of gestation.

This information, along with parallel structural studies (3), indicates a similarity to human lung development and provides fundamental data for future studies on developmentally related pulmonary disorders and accelerated or aberrant pulmonary maturation.

\section{REFERENCES AND NOTES}

1. Adams, F. H., Fujiwara, T., and Latta, D. A.: Alveolar and whole lung phospholipid of premature newborn lambs: Correlation with surface tension, respiratory distress and pathology. Biol. Neonate, 17: 198 (1971).

2. Bartlett, G. R.: Phosphorus assay in column chromatography. J. Biol. Chem., 234: 466 (1959).

3. Boyden, E. A.: The development of the lung in the pigtail monkey (Macaca nemestrina L). Anat. Rec., 186: 15 (1976).

4. Brumley, G. W., Chernick, V., Hodson, W. A., Normand, C., Fenner, A. and Avery, M. E.: Correlation of mechanical stability, morphology, pulmonary surfactant and phospholipid content in the developing lamb lung. J. Clin. Invest., 46: 863 (1967).

5. Buckingham, S., and Avery, M. E.: The time of appearance of lung surfactant in the fetal mouse. Nature, 193: 699 (1962).

6. Chi, E. Y., Prueitt, J. L., and Lagunoff, D.: Abnormal lamellar bodies in type II pneumocytes and increased lung surface active material in the beige mouse. J. Histochem. Cytochem., 23: 863 (1975).

7. Dittmer, J. C., and Wells, M. A.: Quantitative and qualitative analysis of lipids and lipid components. In: J. M. Lowenstein: Methods in Enzymology, Vol. 14, p. 486 (Academic Press, New York, 1969).
8. Epstein, M. F., and Farrell, P. M.: The choline incorporation pathway: Primary mechanism for de Novo lecithin synthesis in the fetal primate lung. Pediat. Res., 9: 658 (1975).

9. Epstein, M. F., Farrell, P. M., and Chez, R. A.: Fetal lung lecithin metabolism and the amniotic fluid L/S ratio in rhesus monkey gestations. Amer. J. Obstet. Gynecol., 125: 545 (1976).

10. Epstein, M. F., Farrell, P. M., and Chez, R. A.: Lung lecithin synthesis in primates as related to respiratory distress syndrome. In: E. I. Goldsmith and J. Moor-Jakowski: Advances in Medical Primatology, pp. 117-126 (Karger, Basel, 1976).

11. Farrell, P. M., and Avery, M. E.: Hyaline membrane disease. Amer. Rev. Resp. Dis., 111: 657 (1975).

12. Farrell, P. M., Epstein, M. F., Fleischman, A. R., Oakes, G. R., and Chez, R. A.: Lung lecithin biosynthesis in the nonhuman primate fetus: Determination of the primary pathway in vivo. Biol. Neonate, 29: 238 (1976).

13. Frosolono, M. F., Charms, B. L., Pawlowski, R., and Slivka, S.: Isolation, characterization and surface chemistry of a surface active fraction from dog lung. J. Lipid Res., 11: 439 (1970).

14. Gluck, L., Chez, R. A., Kulovich, M. V., Hutchinson, D. L., and Niemann, W. H.: Comparison of phospholipid indicators of fetal lung maturity in the monkey (Macaca mulatta) and baboon (Papio papio). Amer. J. Obstet. Gynecol., 120: 524 (1974).

15. Gluck, L., and Kulovich, M. V.: Lecithin sphingomyelin ratios in amniotic fluid in normal and abnormal pregnancy. Amer. J. Obstet. Gynecol., 115: 539 (1973).

16. Gluck, L., Kulovich, M. V., Borer, R. C., Brenner, P. H., Anderson, G. G., and Spellacy, W. N.: Diagnosis of respiratory distress syndrome by amniocentesis. Amer. J. Obstet. Gynecol., 109: 440 (1971).

17. Gluck, L., Kulovich, M. V., Eidelman, A. E., Cordero, L., and Khazin, A F.: Biochemical development of surface activity in mammalian lung. IV. Pulmonary lecithin synthesis in the human fetus and newborn and etiology of the respiratory distress syndrome. Pediat. Res., 6: 81 (1972).

18. Gluck, L., Motoyama, E. K., Smits, H. L., and Kulovich, M. V.: The biochemical development of surface activity in the mammalian lung. I. The surface active phospholipids: The separation and distribution of surfaceactive lecithin in the lung of the developing rabbit fetus. Pediat. Res., $1: 237$ (1967).

19. Hodson, W. A., Palmer, S., Blakely, G., Murphy, J. H., Woodrum, D. E., and Morgan, T. E.: Lung development in the fetal primate Macaca nemestrina. I. Growth and compositional changes. Pediat. Res., 11: 000 (1977).

20. Hodson, W. A., Suzuki, L., and Bloom, R.: Pulmonary phospholipids in the premature infant. Clin. Res., 18: 223 (1970).

21. Kerr, G. R., and Helmuth, A. C.: Growth and development of the fetal rhesus monkey. V. Fatty acids of phospholipis in fetal lung. Biol. Neonate, 25: 10 (1975).

22. Kerr, G. R., Couture, J., and Allen, J. R.: Growth and development of the fetal rhesus monkey. VI. Morphometric analysis of the developing lung. Growth, 39: 67 (1975).

23. Kikkawa, Y., Motoyama, E. K., and Cook, C. D.: The ultrastructure of the lungs of lambs. Amer. J. Pathol., 47: 877 (1965).

24. King, R. C., and Clemens, J. A.: Surface active materials isolated from dog lung. I. Method of isolation. Amer. J. Physiol., 223: 707 (1972).

25. King, R. C., Ruch, J., Gikas, E. G., Platzker, A. C. G., and Creasy, R. K.: Appearance of apoproteins of pulmonary surfactant in human amniotic fluid. J. Appl. Physiol., 39: 735 (1975).

26. Kling, O. R.: Amniotic fluid assay as an index for gestational age and fetal development in the baboon. Obstet. Gynecol., 43: 704 (1974).

27. Lowry, O. H., Rosebrough, N. J., Farr, A. L., and Randall, R. J.: Protein measurement with the Folin phenol reagent. J. Biol. Chem., 193: 265 (1951).

28. Long, C., and Penny, I. F.: The structure of naturally occurring phosphoglycerides. Biochem. J., 65: 382 (1957).

29. Morgan, T. E., and Edmunds, L. H., Jr.: Pulmonary artery occlusion. III. Biochemical alterations. J. Appl. Physiol., 22: 1012 (1967).

30. Morgan, T. E., Finley, T. N., and Fialkow, H.: Comparison of the composition and surface activity of "alveolar" and whole lung lipids in the dog. Biochem. Biophys. Acta., 106: 403 (1965).

31. Murphy, J., and Luchtel, D.: Lung development in the fetal primate Macaca nemestrina: Ultrastructural changes. (In press.)

32. Murphy, J., Palmer, S., Prueitt, J., Yount, J., Standaert, T., Morgan, T., and Hodson, W. A.: Hyaline membrane disease in the premature monkey. Amer. Rev. Resp. Dis., 113: 44 Suppl. (1974).

33. Novy, M. J., Portman, O. W., and Bell, M.: Evidences for pulmonary and other sources of amniotic fluid phospholipids in the rhesus monkey. In: C. A. Villee, D. B. Villee, and J. Zuckerman: Respiratory Distress Syndrome, pp. 205-218 (Academic Press, New York, 1973).

34. Orzalesi, M. M., Motoyama, E. K., Jacobson, H. N., Kikkawa, Y., Reynolds, E. O. R., and Cook, C. D.: The development of the lungs of lambs. Pediatrics, 35: 375 (1965).

35. Parker, F., and Peterson, N. D.: Quantitative analysis of phospholipid and phospholipid fatty acids from silica gel thin-layer chromatograms. J. Lipid Res., 6: 455 (1965).

36. Pawlowski, R., Frosolono, M. F., Charms, B. L., and Przybylski, R.: Intraand extra-cellular compartmentalization of the surface active fraction in dog lung. J. Lipid Res., 21: 538 (1971).

37. Platzker, A. C. K., Kitterman, J. A., Mesher, E. J., Clements, J. A., and Tooley, W. H.: Surfactant in the lung and tracheal fluid of the fetal lamb and acceleration of its appearance by dexamethasone. Pediatrics., 56: 544 (1975).

38. Stolte, L. A. M.: Pregnancy in the rhesus monkey. In: G. H. Bourne: The 
Rhesus Monkey, Vol. II, p. 216 (Academic Press, New York, 1975).

39. Ways, P. O., Reed, C. F., and Hanahan, D. J.: Red cell and plasma lipids in acanthocytosis. J. Clin. Invest., 42: 1248 (1963).

40. Weinhold, P. A., and Villee, C. A.: Phospholipid metabolism in the liver and lung of rats during development. Biochem. Biophys. Acta, J06: 540 (1965).

41. The present address of Dr. T. E. Morgan is: Division of Biomedical Research, Association of American Medical Colleges, Suite 200, One Dupont Circle N.W., Washington, D.C. 20036 (USA).

Copyright (C) 1977 International Pediatric Research Foundation, Inc.
42. This research was supported by Grants HD-0057, HD-00747, RR00166, and Pulmonary SCOR HL-14152 from the National Institutes of Health.

43. Requests for reprints should be addressed to: W. Alan Hodson, M.D., Department of Pediatrics, University of Washington, Seattle, Wash. 98195 (USA).

44. Received for publication October $21,1976$.

45. Accepted for publication February 23, 1977

Pediat. Res. 11: 1021-1026 (1977)

Bilirubin neonate

jaundice phototherapy

\title{
Effect of Intermittent Phototherapy on Bilirubin Dynamics in Gunn Rats
}

\author{
THOMAS P. VOGL, ${ }^{(20)}$ HOWARD CHESKIN, THOMAS A.BLUMENFELD, WILLIAM T. SPECK, \\ AND M. RICHARD KOENIGSBERGER \\ Department of Pediatrics, Columbia University College of Physicians and Surgeons, New York, New York, USA
}

Summary

To determine whether continuous phototherapy is necessary to control neonatal jaundice, groups of jaundiced (Gunn) rats were exposed to four blue light regimens: continuous light, 30 $\mathrm{min}$ light/30 min dark, $6 \mathrm{~min}$ light $/ 6 \mathrm{~min}$ dark, and $6 \mathrm{~min}$ light $/ 18$ min dark. An exponential decrease in serum bilirubin concentration (SBC) was found with all regimens. A logarithmic dose response curve was obtained with a $50 \%$ light dose exhibiting $\mathbf{7 5} \%$ efficacy, and a $25 \%$ light dose exhibiting $59 \%$ efficacy compared to continuous phototherapy. The time constants of the decrease in serum bilirubin concentration also varied logarithmically with doses with continuous therapy having a time constant of 1.4 days, and $6 \mathrm{~min}$ light/18 $\mathrm{min}$ dark therapy having a time constant of 3 days.

\section{Speculation}

In view of the concern which has been expressed over the potential long term side effects of phototherapy, the present study suggests that intermittent phototherapy, with a light cycle of approximately 30-60 min duration, and a concomitant dosage decrease of 50-75\% may effectively control neonatal hyperbilirubinemia.

The rate-limiting step of a major component of the mechanisms of phototherapy of hyperbilirubinemia may be the diffusion of bilirubin from blood to sites in the skin or the associated diffusion of bilirubin photoproducts out of the skin into the blood $(6,14)$. These diffusion processes occur slowly relative to the rapid $\left(<10^{-8} \mathrm{sec}\right)$ photochemical reaction of light with bilirubin $(3,8)$. We postulate that the light dosage inherent in continuous phototherapy may exceed that necessary to lower serum bilirubin concentration in neonatal jaundice and hypothesize that intermittent phototherapy may be equally as effective as continuous illumination. This study evaluates the effectiveness of three intermittent phototherapy schedules in reducing the serum bilirubin concentration of adult homozygous Gunn rats.

\section{MATERIALS AND METHODS}

Dorsally shaved adult homozygous Gunn rats were used for all experiments and phototherapy was administered using six Westinghouse Special Blue narrow spectrum fluorescent lamps (no. F20T12BB, Westinghouse Electric Company, Pittsburgh, Pa.). At the level of the rats these lamps produced a radiant flux (irradiance) of $0.014 \mathrm{~mW} / \mathrm{cm}^{2}$ at $310-390 \mathrm{~nm} ; 1.0 \mathrm{~mW} / \mathrm{cm}^{2}$ at $410-500 \mathrm{~nm} ; 0.15 \mathrm{~mW} / \mathrm{cm}^{2}$ at $500-565 \mathrm{~nm}$; and $0.045 \mathrm{~mW} / \mathrm{cm}^{2}$ at $635-700 \mathrm{~nm}$, as measured with a light meter constructed by one of us and calibrated against a fluorescent lamp at the National Bureau of Standards $(1,16)$.

\section{CONTINUOUS PHOTOTHERAPY}

To determine the effect of continuous phototherapy an experimental group of six rats was studied during three 10-day periods: period 1, a control period of darkness; period 2, a period of continuous exposure to the phototherapy lights; and period 3, a recovery period identical to the control period. A control group of two male and two female rats remained in subdued light ("dark") for 30 days. During the control periods referred to as "dark," the mean irradiances were $0.0008 \mathrm{~mW} / \mathrm{cm}^{2}$ at $310-390$ $\mathrm{nm} ; 0.01 \mathrm{~mW} / \mathrm{cm}^{2}$ at $415-465 \mathrm{~nm} ; 0.01 \mathrm{~mW} / \mathrm{cm}^{2}$ at $500-565$ $\mathrm{nm}$; and $0.005 \mathrm{~mW} / \mathrm{cm}^{2}$ at $635-700 \mathrm{~nm}$ derived primarily from low level, indirect white fluorescent room lights.

\section{INTERMITTENT PHOTOTHERAPY}

Six female experimental rats were studied during seven 10-day periods. These periods were: (1) a control period of darkness, (2) a 30-min light/30-min dark (30L/30D) phototherapy period, (3) a recovery period in the dark, (4) a 6-min light/6-min dark $(6 \mathrm{~L} / 6 \mathrm{D})$ phototherapy period, (5) a recovery period in the dark, (6) a 6-min light/18-min dark $(6 \mathrm{~L} / 18 \mathrm{D})$ phototherapy period, and (7) a recovery period in the dark. Simultaneously, four control rats received no phototherapy; however, on the last day of the second recovery period (period 5) one control rat died.

At the beginning of each experimental cycle, after $8 \mathrm{hr}$, and 